\title{
Reliability Analysis for Two Components Connected in Parallel with Lindley Probability Model
}

\author{
Ehtesham Hussain ${ }^{1, *}$ and Masood ul $\mathrm{Haq}^{2}$ \\ ${ }^{1}$ Department of Statistics, University of Karachi, Karachi, Pakistan \\ ${ }^{2}$ Usman Institute of Technology, Karachi, Pakistan
}

\begin{abstract}
Reliability of structures has been discussed by several authors using probability models. Some of the early researches have been discussed by Birnbaum (1956) [1] in which two independent variables $X$ and $Y$ are defined as "Strength" and "Stress" respectively.

This research is an extension of Mann- Whitney paper (1947) [2] on $P(Y<X)$. Beg (1979c, 1980b, 1980c) [3-5] estimated reliability i.e $R=P(Y<X)$, by taking two parameter Pareto and Power function distributions. Gupta and Gupta (1990) [6] have found point estimates of $\mathrm{R}=\mathrm{P}(\mathrm{aX}>\mathrm{bY})$ by Maximum likelihood and MVUE of $\mathrm{R}$. In the present paper we have considered $R=P(Y<X)$ where $X$ and $Y$ independently follow Lindley distribution. The MLE and Moment estimators of the distribution and then that of $\mathrm{R}$ have been found. A simulation study has been done to estimate biasedness and Confidence interval of $R$.
\end{abstract}

Keywords: Reliability $\mathrm{R}=\mathrm{P}(\mathrm{Y}<\mathrm{X})$, Lindley distribution, Maximum Likelihood Estimator, Moment Estimator.

\section{INTRODUCTION}

In real life situations, Reliability has been discussed in several ways. For example let $Y$ represent the Maximum pressure in a chamber which is generated by ignition of a chemical, and let $X$ represent the Strength of the Chamber, then the reliability of the chamber can be stated by the probability expression: $R=P(Y<X)$. Besides, the Reliability of the entire system which consists of several components is called the System Reliability. It is the probability that the system does not fail upto a certain time instant. Two particular cases are: When the components are connected in Series and when these are connected in parallek.

Al-Mutairi, M.E. Ghitany \& D. Kundu, (2010) [7] have discussed the Asymtotic distribution of the MLE while discussing $\mathrm{P}(\mathrm{Y}<\mathrm{X})$ in Stress-Strength problems.

The series system works when each component in the system works. The Reliability of the system then depends on the reliability of each of its components. Thus the system reliability is found by the Minimum value of reliability that some component $R$ is having.

The parallel system works when at least one of its component works. Therefore the reliability of such a system is found from the Maximum value of reliability of a component.

*Address correspondence to this author at the Department of Statistics, University of Karachi, Karachi, Pakistan; Tel: (9921)-9926 1300-7/ext: 2298; Fax: 99261340, 99261330; E-mail: ehussain@uok.edu.pk

\section{THEORY AND METHODS}

First we discuss the Reliability of a series system. Suppose there are " $n$ " components in the system. Let

$A_{i}=i$ th. component is working, and

$R_{i}=$ Re liability of ith.system

Thus reliability of the system is:

$R_{s}=$ Probability $($ All components working)

$=P\left(A_{1} \cap A_{2} \cap \ldots \cap A_{n}\right)$

$=P\left(A_{1}\right) P\left(A_{2}\right) \cdots P\left(A_{n}\right)$ assuming independence.

$=\prod_{i=1}^{n} R_{i}$ (Assuming independence)

When there are $\mathrm{n}$ components and these are connected in parallel, the expression of reliability is:

$$
\begin{aligned}
& R_{p}=P\left(A_{1} \cup A_{2} \cup \ldots A_{n}\right) \\
& =P\left(A_{1}^{\prime} \cap A_{2}^{\prime} \cap \ldots A_{n}^{\prime}\right) \\
& =1-P\left(A_{1}^{\prime} \cap A_{2}^{\prime} \cap \ldots A_{n}^{\prime}\right)
\end{aligned}
$$

Therefore:

$$
R_{p}=1-\prod_{i=1}^{n}\left(1-R_{i}\right)
$$

\section{SELECTION AND MODELLING}

Here we have Stress $(\mathrm{X})$ And Strength $(\mathrm{Y})$ as two independent continuous variables, each following a Lindley distribution with different parameters as follows: 


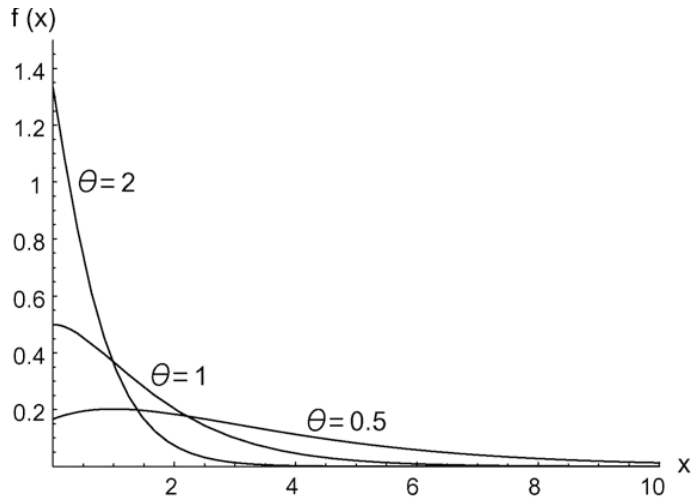

Figure 1: The Lindley pdf for selected parameters.

The probability functions for parameters "a", and "b" are:

$f(x)=\frac{a^{2}}{(1+a)}(1+x) e^{-a x} ; x \geq 0, a>0$

$f(y)=\frac{b^{2}}{(1+b)}(1+y) e^{-b y}, y \geq 0, b>0$

For a single component the reliability is:

$\mathrm{R}=$ Probability (the component performs satisfactorily)

$P($ Stress $<$ Strength $)$

$P(y<x)$

$=\int_{-\infty}^{\infty} \int_{-\infty}^{x} f(x, y) d y d x$

where integrand is the joint density function of $x$ and $y$.

$=\int_{-\infty}^{\infty} F_{y}(x) f(x) d x$

where $F_{y}(x)=\int_{-\infty}^{x} f(y) d y$

Table 2: $P(Y<X)$ for selected parameter values of $(\mathbf{a}, \mathbf{b})$

$$
\begin{aligned}
& F_{y}(x)=\int_{-\infty}^{x} \frac{b^{2}}{(1+b)}(1+y) e^{-b y} d y \\
& =1-e^{-b x}-\frac{b}{(1+b)} x e^{-b x}
\end{aligned}
$$

Substituting the expression (8) in (7), Reliability R is:

$$
\begin{aligned}
& R=\int_{-\infty}^{+\infty}\left[1-e^{-b x}-\frac{b}{(1+b)} x e^{-b x}\right] \frac{a^{2}}{(1+a)}(1+x) e^{-a x} d x \\
& R=\frac{a^{2}\left[a(1+a)+(1+a)(3+a) b+(3+2 a) b^{2}+b^{3}\right]}{(1+a)(1+b)(a+b)^{3}}
\end{aligned}
$$

SECTION IV: Computation of Exact Reliability in a single component.

The exact values of Reliabilities are obtained when the values of the parameters "a" and " $b$ " are specified. In order to be a little brief, we have selected only a few values of "a" and also of "b". The values of "a" and "b" are given in the following Table 1:

Table 1:

\begin{tabular}{|c|c|c|c|c|c|c|c|}
\hline $\mathrm{a}$ & 0.5 & 1.0 & 1.5 & 2.0 & 2.5 & 3.0 & 3.5 \\
\hline $\mathrm{b}$ & 0.5 & 1.0 & 1.5 & 2.0 & 2.5 & 3.0 & 3.5 \\
\hline
\end{tabular}

With the above values of "a" and " $b$ ", the following table of Reliability of a component is calculated using expression (9):

The Exact values of Reliabilities i.e $R=P(Y \leq X)$ show that for each pair of value of $(a, b)$, the value of $R$ continues to decrease as the value of "a" gradually increases, after starting at a certain value while keeping "b" at a fixed level. It also shows that for $a=$ 0.5 ,"b $=3.5$ " the $\mathrm{R}$ value attains a high value of 0.9357 or more than $93 \%$.

\begin{tabular}{|c|c|c|c|c|c|c|c|}
\hline \multicolumn{7}{|c|}{ Stress Parameter "b" } \\
\hline Strength parameter "a" $\downarrow$ & $\mathbf{0 . 5}$ & $\mathbf{1 . 0}$ & $\mathbf{1 . 5}$ & $\mathbf{2 . 0}$ & $\mathbf{2 . 5}$ & $\mathbf{3 . 0}$ & $\mathbf{3 . 5}$ \\
\hline \hline 0.5 & 0.5 & 0.728395 & 0.825000 & 0.874667 & 0.903880 & 0.922741 & 0.935764 \\
\hline 1.0 & 0.271605 & 0.5 & 0.633600 & 0.716049 & 0.770512 & 0.808594 & 0.836458 \\
\hline 1.5 & 0.175000 & 0.366400 & 0.50000 & 0.592420 & 0.658482 & 0.707407 & 0.7448 \\
\hline 2.0 & 0.125333 & 0.283951 & 0.407580 & 0.5 & 0.569926 & 0.624000 & 0.66675 \\
\hline 2.5 & 0.0961199 & 0.229488 & 0.341518 & 0.430074 & 0.5 & 0.555919 & 0.601337 \\
\hline 3.0 & 0.0772595 & 0.191406 & 0.292593 & 0.376000 & 0.444081 & 0.5 & 0.546427 \\
\hline 3.5 & 0.0642361 & 0.163542 & 0.255200 & 0.333250 & 0.398663 & 0.453573 & 0.5 \\
\hline
\end{tabular}




\section{SAMPLING PROPERTIES OF RELIABILITIES (ONE COMPONENT)}

In order to study the sampling properties of Reliabilities, we use the Maximum Likelihood Estimator of R, i.e. $\hat{R}=R(\hat{a}, \hat{b}) \quad$ where

$\hat{a}=\frac{(1-\bar{x})+\sqrt{\bar{x}^{2}+6 \bar{x}+1}}{2 \bar{x}}$ and $\hat{b}=\frac{(1-\bar{y})+\sqrt{\bar{y}^{2}+6 \bar{y}+1}}{2 \bar{y}}$

A simulation study has been conducted, based on parametric bootstrap to investigate the performance of the estimator $\hat{R}$. This is for one component performance regarding Reliability.

The simulation was initiated by first selecting values of "a" and "b" and sample sizes $(\mathrm{n}=\mathrm{m}=$ $10,15,20,25,30)$.

For each pair of $[a, b]$ and sample sizes, as given above, 1000 bootstrap samples of $\mathrm{X}$ and $\mathrm{Y}$ are generated. These give 1000 Maximum Likelihood Estimates of "a" and "b" i.e $\hat{a}^{*}$ and " $\hat{b}^{*} "$ respectively. Using these estimators we get 1000 MLE bootstrap estimates of $\mathrm{R}$ i.e $\hat{R}^{*}, \hat{R}_{1}^{*}, \ldots \ldots . ., \hat{R}_{1000}^{*}$.

The 1000 bootstrap estimates of $R$ are used to obtain Average

$\hat{R}^{*}$, MSE, and $96 \%$ E fron Percentile Confidence

Intervals for " $R$ " and Centre of Confidence Interval

The results are presented in the following Table $\mathbf{3}$, which is only for sample size 10 for being brief. The tables for other sample sizes are computed by the author.

Table 3: True values of $R$; Average, MSE, Lower $L$, Upper L, Bootstrap results for $m=30, n=30, B$ $=1000$

\begin{tabular}{|c|c|c|c|c|c|}
\hline S. No & $\mathbf{R}$ & $E\left(\hat{R}^{*}\right)$ & MSE & Lower L & Upper L \\
\hline \hline 1 & 0.87466 & 0.87236 & 0.00100 & 0.80168 & 0.92420 \\
\hline 2 & 0.90388 & 0.90178 & 0.00069 & 0.84640 & 0.94182 \\
\hline 3 & 0.922741 & 0.92038 & 0.00050 & 0.86652 & 0.95358 \\
\hline 4 & 0.935764 & 0.93319 & 0.00034 & 0.88885 & 0.96148 \\
\hline 5 & 0.945222 & 0.94325 & 0.00025 & 0.90812 & 0.96793 \\
\hline 6 & 0.808594 & 0.80539 & 0.00194 & 0.71159 & 0.88106 \\
\hline 7 & 0.836458 & 0.83180 & 0.00172 & 0.74446 & 0.89979 \\
\hline 8 & 0.857600 & 0.85412 & 0.00121 & 0.78436 & 0.91348 \\
\hline 9 & 0.59242 & 0.59139 & 0.00448 & 0.45521 & 0.71651 \\
\hline 10 & 0.658482 & 0.65354 & 0.00367 & 0.52509 & 0.76306 \\
\hline
\end{tabular}

TWO COMPONENTS CONNECTED IN PARALLEL

Consider two identical components, connected in parallel, and for each component Stress is denoted by $Y$ and strength of components $X_{1}, X_{2}$ and each follows a Lindley distribution. Then the Reliability of the System is: (The Integration in expression 11 below has been done by MATHEMATIKA)

$$
\begin{aligned}
R_{2 p}= & P\left(Y<\max \left(X_{1}, X_{2}\right)\right) \\
= & 1-\int_{-\infty}^{\infty}[F(Y)]^{2} d F(Y) \\
& 2 a^{4}\left\{8 a^{5}(1+b)+4 a^{4}(4+7 b(2+b))\right. \\
& +2 a^{3}(4+b(44+19 b(3+b))) \\
& \left.+5 a^{2} b(26+5 b(4+b))\right) \\
& +a b^{2}(45+b(75+8 b(5+b))) \\
= & \frac{\left.\left.\left.+b^{2}(15+b(6+b))\right)\right)\right)}{(1+b)(a+b)^{3}(2 a+b)^{4}}
\end{aligned}
$$

In order to obtain the Maximum Likelihood Estimator (MLE) of $R_{2 p}$ i.e $\hat{R}_{2 p}$, the MLE's of "a" and "b" are substituted in the expression of $R_{2 p}$ in (!!).

The MLE's of "a" and "b" are shown in (10). Thus $\hat{R}_{2 p}=R_{2 p}(\hat{a}, \hat{b})$.

As the expression of $\hat{R}_{2 p}$ is quite complicated, a Bootstrap simulation has been applied with the following sample sizes:

$(m, m)=(10,10),(15,15),(20,20),(25,25),(30,30)$ and selected the following values of "a", "b" :

$a=0.5,1.0,1.5,2.0$, where "a" is in the pdf of $X$ or Strength.

$b=2.0,2.5,3.0,4.0$, , where "b" is in the pdf of $Y$ or Stress

For each pair of $(a, b), 1000$ Bootstrap sample estimates of $\hat{R}_{2 p}^{*}$ are generated for sample sizes (m, $\mathrm{m})$. Then Averages of the Estimators are calculated by

$\hat{R}_{2 p}=\frac{1}{1000} \sum_{i=1}^{1000} \hat{R}_{\mathfrak{2}_{p}}^{*} ; \quad$ Mean Sq. Error $=$ $\frac{1}{1000} \sum_{i=1}^{1000}\left(\hat{R}_{2 p i}{ }^{*}-\hat{R}_{2 p}\right)^{2}$

In the present paper, $(m, m)=(10,10)$ and only one value of the parameter "a" i.e 0.5 and one of "b" i.e.2 has been shown in the table. The complete table is in the thesis. 
Table 4: True value, Av. value, mse, conf.limit $L$, conf.limit U of conf.interval

\begin{tabular}{|c|c|c|c|c|c|}
\hline & $R_{2 p}$ & $E\left(\hat{R}_{2 p}^{*}\right)$ & MSE & $\mathbf{L}$ & $\mathbf{U}$ \\
\hline \hline 1 & 0.970184 & 0.961127 & 0.00105926 & 0.872452 & 0.995311 \\
\hline 2 & 0.981881 & 0.974643 & 0.000522964 & 0.917672 & 0.997364 \\
\hline 3 & 0.988059 & 0.982593 & 0.000292415 & 0.937800 & 0.998373 \\
\hline 4 & 0.991643 & 0.987439 & 0.000148898 & 0.955938 & 0.998647 \\
\hline
\end{tabular}

The above Table 4 shows that the Average value remains within the Lower $(\mathrm{L})$ limit and Upper $(\mathrm{U})$ limit for the selected values of parameter values.

\section{CONCLUSION}

In this paper we have calculated the Reliability of a system, when the components of the system are connected in parallel and the probability model of each component follows the Lindly model. The study has been done theoretically for a single component, and then for two components when connected in parallel.
Afterwards, a Simulation study has been performed to confirm the theoretical results.

\section{REFERENCES}

[1] Birnbaum ZW. On a use of Mann-Whitney statistics. Proc. Third Berkeley Symp. in Math. Statist. Probab., University of California Press 1956; Vol. 1: pp. 13-17.

[2] Mann $\mathrm{H}$, Whitney D. On a test whether one of two random variables is stochastically larger than the other. Ann Math Stat 1947; 18: 50-60.

[3] Beg MA. Estimation of $\operatorname{Pr}(Y<X)$ for the Pareto distribution. IEEE Trans. Reliability R-28, 1979c; 411.

[4] Beg MA. On the estimation of $\mathrm{P}(\mathrm{Y}<\mathrm{X})$ for the two-parameter exponential distribution. Metrika 1980b; 27: 29-34. http://dx.doi.org/10.1007/BF01893574

[5] Beg MA. Estimation of $\operatorname{Pr}(\mathrm{Y}<\mathrm{X})$ for truncation parameter distri-bution Communication in Statistics: Theory and methods 1980c; 9(3): 327-345.

[6] Gupta RD, Gupta RC. Estimation of $\operatorname{Pr}\left(a^{\prime} x>b^{\prime} y\right)$ in the multivariate normal case. Statistics 1990; 21(1): 91-97.

[7] Al-Mutairi ME, Ghitany, Kundu D. Inferences on StressStrength Reliability from Lindley Distribution 2010.

Received on 25-04-2015

Accepted on 02-05-2015

Published on 21-05-2015

http://dx.doi.org/10.6000/1929-6029.2015.04.02.5

(c) 2015 Hussain and Haq; Licensee Lifescience Global.

This is an open access article licensed under the terms of the Creative Commons Attribution Non-Commercial License (http://creativecommons.org/licenses/by-nc/3.0/) which permits unrestricted, non-commercial use, distribution and reproduction in any medium, provided the work is properly cited. 